\title{
Global and Blow-Up Solutions for Nonlinear Hyperbolic Equations with Initial-Boundary Conditions
}

\author{
Ülkü Dinlemez ${ }^{1}$ and Esra Aktaş ${ }^{2}$ \\ ${ }^{1}$ Department of Mathematics, Faculty of Science, Gazi University, Teknikokullar, Ankara, Turkey \\ ${ }^{2}$ Incirli Mahallesi, Karaelmas Sokak, Yunusemre Caddesi 51/18, Incirli, Ankara, Turkey \\ Correspondence should be addressed to Ülkü Dinlemez; ulku@gazi.edu.tr
}

Received 24 December 2013; Revised 7 March 2014; Accepted 20 March 2014; Published 13 April 2014

Academic Editor: D. D. Ganji

Copyright (C) 2014 Ü. Dinlemez and E. Aktaş. This is an open access article distributed under the Creative Commons Attribution License, which permits unrestricted use, distribution, and reproduction in any medium, provided the original work is properly cited.

We consider an initial-boundary value problem to a nonlinear string equations with linear damping term. It is proved that under suitable conditions the solution is global in time and the solution with a negative initial energy blows up in finite time.

\section{Introduction}

We study the damped nonlinear string equation with source term $|u|^{\alpha} u$ :

$$
\begin{array}{r}
u_{t t}+u_{t}=\left(\sigma\left(\left|u_{x}\right|^{2}\right) u_{x}\right)_{x}+|u|^{\alpha} u, \\
(x, t) \in(0,1) \times[0, T],
\end{array}
$$

where $1<\alpha, \sigma(s)$ is a smooth function for $0 \leq s$ with the initial conditions

$$
u(x, 0)=u_{0}(x), \quad u_{t}(x, 0)=u_{1}(x), \quad x \in[0,1],
$$

and boundary conditions

$$
\begin{gathered}
u(1, t)=0, \quad t \in(0, T), \\
\sigma\left(\left|u_{x}(0, t)\right|^{2} u_{x}(0, t)\right)-u_{t}(0, t)=2 \phi(t), \quad t \in[0, T], \\
\sigma\left(\left|u_{x}(0, t)\right|^{2} u_{x}(0, t)\right)+u_{t}(0, t)=2 \psi(t), \quad t \in[0, T] .
\end{gathered}
$$

The problem (1)-(3) can be regarded as modelling a nonlinear string with vertical displacement function $u(x, t)$ in $\mathbb{R}$. And this problem has nonlinear mechanical damping of the form $|u|^{\alpha} u$. The right end of the string makes it steady. The input $\phi(t)$ function and the output $\psi(t)$ function are applied on the left.

$\mathrm{Wu}$ and $\mathrm{Li}[1]$ studied the motion for a nonlinear beam model with nonlinear damping $a\left|\phi_{t}\right|^{m-1} \phi_{t}$ and external forcing $b|\phi|^{p-1} \phi$ terms. They showed that this model has a unique global solution and blow-up solution under the same conditions. Levine et al. [2] and Levine and Serrin [3] studied abstract version. Georgiev and Todorova [4] studied nonlinear wave equations involving the nonlinear damping term $\left|u_{t}\right|^{m-1} u_{t}$ and source term of type $\left|u_{t}\right|^{p-1} u_{t}$. They proved global existence theorem with large initial data for $1<p \leq m$. Hao and Li [5] studied the global solutions for a nonlinear string with boundary input and output. Dinlemez [6] proved the global existence and uniqueness of weak solutions for the initial-boundary value problem for a nonlinear wave equation with strong structural damping and nonlinear source terms in $\mathbb{R}$. A lot of papers in connection with blowup, global solutions and existence of weak solutions were studied in [7-15].

In this paper we first find energy equation for the problem (1)-(3). Then we prove the solutions of the problem (1)-(3) are global in time under some conditions on the function $\sigma(s)$, input $\phi(t)$, and the output $\psi(t)$. Finally we establish a blow-up result for solutions with a negative initial energy. Our approach is similar to the one in [5].

\section{Main Results}

Now we give the following lemma for energy equation for the problem (1)-(3). 
Lemma 1. Let $1<\alpha$ and $u(x, t)$ be a solution of the problem (1)-(3). Then the energy equation of the problem (1)-(3) is

$$
\begin{gathered}
E(t)=\frac{1}{2}\left\|u_{t}\right\|_{2}^{2}-\frac{1}{\alpha+2}\|u\|_{\alpha+2}^{\alpha+2}+\frac{1}{2} \int_{0}^{1} \int_{0}^{\left|u_{x}\right|^{2}} \sigma(\xi) d \xi d x, \\
\frac{d}{d t} E(t)=\phi^{2}(t)-\psi^{2}(t)-\left\|u_{t}\right\|_{2}^{2} .
\end{gathered}
$$

Proof. Multiplying (1) with $u_{t}$ and integrating over $(0,1)$, then we get

$$
\begin{aligned}
& \frac{d}{d t}\left\{\frac{1}{2}\left\|u_{t}\right\|_{2}^{2}-\frac{1}{\alpha+2}\|u\|_{\alpha+2}^{\alpha+2}\right\} \\
& \quad=\int_{0}^{1}\left(\sigma\left(\left|u_{x}\right|^{2}\right) u_{x}\right)_{x} u_{t} d x-\left\|u_{t}\right\|_{2}^{2} .
\end{aligned}
$$

Applying integration by parts in the right hand side of (6), we find

$$
\begin{aligned}
\int_{0}^{1}\left(\sigma\left(\left|u_{x}\right|^{2}\right) u_{x}\right)_{x} u_{t} d x= & -\sigma\left(\left|u_{x}(0, t)\right|^{2}\right) u_{x}(0, t) u_{t}(0, t) \\
& -\frac{1}{2} \frac{d}{d t} \int_{0}^{1} \int_{0}^{\left|u_{x}\right|^{2}} \sigma(\xi) d \xi d x
\end{aligned}
$$

And using boundary conditions in equality (7), we obtain

$$
\begin{gathered}
\frac{d}{d t}\left\{\frac{1}{2}\left\|u_{t}\right\|_{2}^{2}-\frac{1}{\alpha+2}\|u\|_{\alpha+2}^{\alpha+2}+\frac{1}{2} \int_{0}^{1} \int_{0}^{\left|u_{x}\right|^{2}} \sigma(\xi) d \xi d x\right\} \\
=\phi^{2}(t)-\psi^{2}(t)-\left\|u_{t}\right\|_{2}^{2} .
\end{gathered}
$$

Hence the proof is completed.

Next we give the following theorem for global solutions in time.

Theorem 2. Assume that $u(x, t)$ is a solution of the problem (1)-(3) with $1<\alpha$ and

(i) $\sigma(s)$ satisfies the following condition:

$$
|s|^{\alpha} \leq \sigma(s), \quad \text { for } s \in \mathbb{R}^{+} \cup\{0\},
$$

(ii) the input and the output functions satisfy

$$
\phi^{2}(t) \leq \psi^{2}(t)
$$

Then the solution $u(x, t)$ is global in time.

Proof. Let

$$
\begin{aligned}
G(t) & :=E(t)+\frac{2}{\alpha+2}\|u\|_{\alpha+2}^{\alpha+2} \\
& =\frac{1}{2}\left\|u_{t}\right\|_{2}^{2}+\frac{1}{2} \int_{0}^{1} \int_{0}^{\left|u_{x}\right|^{2}} \sigma(\xi) d \xi d x+\frac{1}{\alpha+2}\|u\|_{\alpha+2}^{\alpha+2} .
\end{aligned}
$$

Differentiating $G(t)$ with respect to $t$ and using (5), we get

$$
\frac{d}{d t} G(t)=\phi^{2}(t)-\psi^{2}(t)-\left\|u_{t}\right\|_{2}^{2}+2 \int_{0}^{1}|u|^{\alpha} u u_{t} d x .
$$

Using the Cauchy-Schwarz inequality in the last term of (12), we obtain

$$
\begin{aligned}
2 \int_{0}^{1}|u|^{\alpha} u u_{t} d x & \leq 2 \int_{0}^{1}|u|^{\alpha+1}\left|u_{t}\right| d x \\
& \leq\|u\|_{2(\alpha+1)}^{2(\alpha+1)}+\left\|u_{t}\right\|_{2}^{2}
\end{aligned}
$$

and it follows from (12), (13), and (10) that we have

$$
\frac{d}{d t} G(t) \leq\|u\|_{2(\alpha+1)}^{2(\alpha+1)}+\left\|u_{t}\right\|_{2}^{2} .
$$

By assumption (9) and integrating over $\left(0,\left|u_{x}\right|^{2}\right)$ and $(0,1)$, respectively, we yield

$$
\frac{1}{\alpha+1}\left\|u_{x}\right\|_{2(\alpha+1)}^{2(\alpha+1)} \leq \int_{0}^{1} \int_{0}^{\left|u_{x}\right|^{2}} \sigma(\xi) d \xi d x .
$$

Furthermore, we have

$$
\begin{aligned}
|u(x, t)|^{2(\alpha+1)} & =\left|\int_{x}^{1} u_{\xi}(\xi, t) d \xi\right|^{2(\alpha+1)} \\
& \leq \int_{x}^{1}\left|u_{\xi}(\xi, t)\right|^{2(\alpha+1)} d \xi \\
& \leq \int_{0}^{1}\left|u_{x}(x, t)\right|^{2(\alpha+1)} d x=\left\|u_{x}(x, t)\right\|_{2(\alpha+1)}^{2(\alpha+1)},
\end{aligned}
$$

and then

$$
\|u(x, t)\|_{2(\alpha+1)}^{2(\alpha+1)} \leq\left\|u_{x}(x, t)\right\|_{2(\alpha+1)}^{2(\alpha+1)} .
$$

Combining (11), (14), (15), and (17), we get

$$
\frac{d G(t)}{d t} \leq \frac{1}{\xi_{1}} G(t),
$$

where $\xi_{1}=\min \{1 / 2,1 /(\alpha+1)\}$. Using Gronwall's inequality, we have

$$
G(t) \leq G(0) e^{\left(1 / \xi_{1}\right) t} .
$$

Therefore together with the continuation principle and the definition of $G(t)$ we complete the proof of Theorem 2 .

Then we give the following theorem for the blow-up solutions of the problem (1)-(3).

Theorem 3. Let $u(x, t)$ be a solution of the problem (1)-(3) with $1<\alpha$. Assume that

(i) there exists $1<\varepsilon<(\alpha+2) / 2$ such that the function $\sigma(s)$ satisfies

$$
\sigma(s) s \leq \frac{\varepsilon}{2} \int_{0}^{s} \sigma(\zeta) d \zeta \quad \text { for } s \in \mathbb{R}^{+} \cup\{0\},
$$


(ii) the initial values satisfy

$$
E(0) \leq 0, \quad 0<\int_{0}^{1} u_{0}(x) u_{1}(x) d x
$$

(iii) the input and output functions satisfy

$$
\begin{aligned}
& \phi^{2}(t) \leq \psi^{2}(t), \\
& (\psi(t)+\phi(t))\left(\int_{0}^{t}(\psi(s)-\phi(s)) d s+u_{0}(0)\right) \leq 0,
\end{aligned}
$$

(iv) $u(x, t)$ satisfies $1 \leq\|u\|$.

Then the solution $u(x, t)$ blows up in finite time $T_{\max }$, and

$$
T_{\max } \leq\left(\frac{\alpha+4}{\alpha \eta}\right) N^{-\alpha /(\alpha+4)}(0),
$$

where $\eta$ is some positive constant independent of the initial value $\alpha$ and $N(t)$ are given by (25).

Proof. We define

$$
\begin{gathered}
M(t):=-E(t), \quad \gamma:=\frac{\alpha}{2(\alpha+2)}, \\
N(t):=M^{1-\gamma}(t)+\int_{0}^{1} u(x, t) u_{t}(x, t) d x .
\end{gathered}
$$

By virtue of (5), (21), (22), and (24), we get

$$
\begin{gathered}
\frac{d M(t)}{d t}=\left\|u_{t}\right\|_{2}^{2}+\psi^{2}(t)-\phi^{2}(t) \geq 0, \\
0 \leq M(0) \leq M(t), \quad \text { for } 0 \leq t .
\end{gathered}
$$

Taking a derivative of (25) and using (26), we have

$$
\begin{aligned}
& \frac{d N(t)}{d t} \\
& \quad=(1-\gamma) M^{-\gamma}(t) M^{\prime}(t)+\int_{0}^{1} u_{t}^{2} d x+\int_{0}^{1} u u_{t t} d x \\
& =(1-\gamma) M^{-\gamma}(t)\left(\left\|u_{t}\right\|_{2}^{2}+\psi^{2}(t)-\phi^{2}(t)\right)+\left\|u_{t}\right\|_{2}^{2} \\
& \quad+\int_{0}^{1} u u_{t t} d x .
\end{aligned}
$$

Multiplying (1) by $u$ and integrating over the interval $[0,1]$ and then using boundary conditions (3), we obtain

$$
\begin{aligned}
\int_{0}^{1} u u_{t t} d x & \\
= & \|u\|_{\alpha+2}^{\alpha+2}-\int_{0}^{1} u u_{t} d x-(\psi(t)+\phi(t)) \\
& \times\left(\int_{0}^{t}(\psi(s)-\phi(s)) d s+u_{0}(0)\right) \\
& -\int_{0}^{1} \sigma\left(\left|u_{x}\right|^{2}\right) u_{x}^{2} d x
\end{aligned}
$$

From the definition of $M(t)$ we yield

$$
\begin{aligned}
0= & \varepsilon M(t)+\frac{\varepsilon}{2}\left\|u_{t}\right\|^{2}+\frac{\varepsilon}{2} \int_{0}^{1} \int_{0}^{\left|u_{x}\right|^{2}} \sigma(\xi) d \xi d x \\
& -\frac{\varepsilon}{\alpha+2}\|u\|_{\alpha+2}^{\alpha+2} .
\end{aligned}
$$

Combining (29) and (30) in (28), we get

$$
\begin{aligned}
\frac{d N(t)}{d t}= & (1-\gamma) M^{-\gamma}(t)\left(\left\|u_{t}\right\|_{2}^{2}+\psi^{2}(t)-\phi^{2}(t)\right)+\left\|u_{t}\right\|_{2}^{2} \\
& +\|u\|_{\alpha+2}^{\alpha+2}-\int_{0}^{1} u u_{t} d x-(\psi(t)+\phi(t)) \\
& \times\left(\int_{0}^{t}(\psi(s)-\phi(s)) d s+u_{0}(0)\right) \\
& -\int_{0}^{1} \sigma\left(\left|u_{x}\right|^{2}\right) u_{x}^{2} d x+\varepsilon M(t)+\frac{\varepsilon}{2}\left\|u_{t}\right\|_{2}^{2} \\
& +\frac{\varepsilon}{2} \int_{0}^{1} \int_{0}^{\left|u_{x}\right|^{2}} \sigma(\xi) d \xi d x \\
& -\frac{\varepsilon}{\alpha+2}\|u\|_{\alpha+2}^{\alpha+2} .
\end{aligned}
$$

Using (22) in (31), we obtain

$$
\begin{aligned}
\left(1+\frac{\varepsilon}{2}\right)\left\|u_{t}\right\|_{2}^{2}+\left(\frac{1}{2}-\frac{\varepsilon}{\alpha+2}\right)\|u\|_{\alpha+2}^{\alpha+2}+\frac{1}{2}\|u\|_{\alpha+2}^{\alpha+2} \\
+\int_{0}^{1}\left(\frac{\varepsilon}{2} \int_{0}^{\left|u_{x}\right|^{2}} \sigma(\xi) d \xi-\sigma\left(\left|u_{x}\right|^{2}\right) u_{x}^{2}\right) d x \\
+\varepsilon M(t)-\int_{0}^{1} u u_{t} d x \leq \frac{d N(t)}{d t}
\end{aligned}
$$

Thanks to Young's inequality,

$$
A B \leq \frac{\delta^{p}}{p} A^{p}+\frac{\delta^{-q}}{q} B^{q}, \quad 0 \leq A, B \forall 0<\delta, \frac{1}{p}+\frac{1}{q}=1,
$$

for $\int_{0}^{1} u u_{t} d x$ with $p=q=2$ and $\gamma=2$, and then we get

$$
\int_{0}^{1} u u_{t} d x \leq \int_{0}^{1}\left|u u_{t}\right| d x \leq\left\|u_{t}\right\|^{2}+\frac{1}{4}\|u\|^{2}
$$

From embedding for $L^{p}(0,1)$ and using (iv), we have $\|u\|_{2}^{2} \leq$ $\|u\|_{\alpha+2}^{\alpha+2}$ and putting (34) in (32) we have

$$
\begin{aligned}
\left(1+\frac{\varepsilon}{2}\right)\left\|u_{t}\right\|_{2}^{2}+\left(\frac{1}{2}-\frac{\varepsilon}{\alpha+2}\right)\|u\|_{\alpha+2}^{\alpha+2}+\frac{1}{2}\|u\|_{2}^{2} \\
+\int_{0}^{1}\left(\frac{\varepsilon}{2} \int_{0}^{\left|u_{x}\right|^{2}} \sigma(\xi) d \xi-\sigma\left(\left|u_{x}\right|^{2}\right) u_{x}^{2}\right) d x+\varepsilon M(t) \\
-\left\|u_{t}\right\|_{2}^{2}-\frac{1}{4}\|u\|_{2}^{2} \leq \frac{d N(t)}{d t} .
\end{aligned}
$$


From (20), we get

$$
\varepsilon M(t)+\frac{\varepsilon}{2}\left\|u_{t}\right\|_{2}^{2}+\left(\frac{1}{2}-\frac{\varepsilon}{\alpha+2}\right)\|u\|_{\alpha+2}^{\alpha+2}+\frac{1}{4}\|u\|_{2}^{2} \leq \frac{d N(t)}{d t} .
$$

Choosing $\varepsilon$ and $\kappa=\min \{\varepsilon / 2,(1 / 2-\varepsilon /(\alpha+2)), 1 / 4\}$, we obtain

$$
\kappa\left\{M(t)+\left\|u_{t}\right\|_{2}^{2}+\|u\|_{\alpha+2}^{\alpha+2}+\|u\|_{2}^{2}\right\} \leq \frac{d N(t)}{d t} .
$$

Thanks to (21) and (27), we yield

$$
0<N(0) \leq N(t), \quad \forall 0<t .
$$

Now we estimate $[N(t)]^{1 /(1-\gamma)}$. From Holder's inequality,

$$
\left|\int_{0}^{1} u u_{t} d x\right| \leq\|u\|_{2}\left\|u_{t}\right\|_{2} \leq\|u\|_{\alpha+2}\left\|u_{t}\right\|_{2}
$$

then using Young's inequality again we get

$$
\begin{aligned}
\left|\int_{0}^{1} u u_{t} d x\right| \leq & \frac{\delta^{2(1-\gamma)}}{2(1-\gamma)}\left\|u_{t}\right\|_{2}^{2(1-\gamma)} \\
& +\frac{1-2 \gamma}{2(1-\gamma)} \delta^{-2(1-\gamma) /(1-2 \gamma)}\|u\|_{\alpha+2}^{2(1-\gamma) /(1-2 \gamma)},
\end{aligned}
$$

where $0<\delta$ and $1 / p+1 / q=1$ with $p=2(1-\gamma)$. And so we have

$$
\begin{aligned}
& \left|\int_{0}^{1} u u_{t} d x\right|^{1 /(1-\gamma)} \\
& \leq 2^{1 /(1-\gamma)}\left(\frac{\delta^{2}}{(2(1-\gamma))^{1 /(1-\gamma)}}\left\|u_{t}\right\|_{2}^{2}\right. \\
& \left.\quad+\left(\frac{1-2 \gamma}{2(1-\gamma)}\right)^{1 /(1-\gamma)} \delta^{-2 /(1-2 \gamma)}\|u\|_{\alpha+2}^{2 /(1-2 \gamma)}\right) .
\end{aligned}
$$

Choosing $\beta=\max \left\{\delta^{2} /(1-\gamma)^{1 /(1-\gamma)},((1-2 \gamma) /(1-\gamma))^{1 /(1-\gamma)}\right.$ $\left.\delta^{-2 /(1-2 \gamma)}\right\}$, we obtain

$$
\left|\int_{0}^{1} u u_{t} d x\right|^{1 /(1-\gamma)} \leq \beta\left(\left\|u_{t}\right\|_{2}^{2}+\|u\|_{\alpha+2}^{\alpha+2}\right) .
$$

Therefore we yield

$$
\begin{aligned}
(N(t))^{1 /(1-\gamma)} & \\
& =\left(M^{1-\gamma}(t)+\int_{0}^{1} u(x, t) u_{t}(x, t) d x\right)^{1 /(1-\gamma)} \\
& \leq 2^{1 /(1-\gamma)}\left(M(t)+\left|\int_{0}^{1} u(x, t) u_{t}(x, t) d x\right|^{1 /(1-\gamma)}\right) \\
& \leq C\left(M(t)+\left\|u_{t}\right\|_{2}^{2}+\|u\|_{\alpha+2}^{\alpha+2}+\|u\|_{2}^{2}\right),
\end{aligned}
$$

where $C$ depends on $\delta$ and $\alpha$. From (37) and (43), we have

$$
\eta(N(t))^{1 /(1-\gamma)} \leq \frac{d N(t)}{d t}
$$

where $\eta=\kappa / C$. Integrating $(44)$ over $(0, t)$, then we get

$$
\frac{1}{(N(0))^{-\alpha /(\alpha+4)}-(\alpha /(\alpha+4)) \eta t} \leq(N(t))^{\alpha /(\alpha+4)} .
$$

Hence $N(t)$ blows up in finite time $T_{\max } . T_{\max }$ is given by the inequality as below:

$$
T_{\max } \leq \frac{\alpha+4}{\alpha \eta}(N(0))^{-\alpha /(\alpha+4)}
$$

Consequently the solution blows up in finite time. And the proof of Theorem 3 is now finished.

\section{Conflict of Interests}

The authors declare that there is no conflict of interests regarding the publication of this paper.

\section{Acknowledgment}

The authors would like to thank the referees for the careful reading of this paper and for the valuable suggestions to improve the presentation and style of the paper.

\section{References}

[1] J.-Q. Wu and S.-J. Li, "Global solution and blow-up solution for a nonlinear damped beam with source term," Applied Mathematics, vol. 25, no. 4, pp. 447-453, 2010.

[2] H. A. Levine, P. Pucci, and J. Serrin, "Some remarks on global nonexistence for nonautonomous abstract evolution equations," Contemporary Mathematics, vol. 208, pp. 253-263, 1997.

[3] H. A. Levine and J. Serrin, "Global nonexistence theorems for quasilinear evolution equations with dissipation," Archive for Rational Mechanics and Analysis, vol. 137, no. 4, pp. 341-361, 1997.

[4] V. Georgiev and G. Todorova, "Existence of a solution of the wave equation with nonlinear damping and source terms," Journal of Differential Equations, vol. 109, no. 2, pp. 295-308, 1994.

[5] J. Hao and S. Li, "Global solutions and blow-up solutions for a nonlinear string with boundary input and output," Nonlinear Analysis: Theory, Methods and Applications, vol. 66, no. 1, pp. 131-137, 2007.

[6] Ü. Dinlemez, "Global existence, uniqueness of weak solutions and determining functionals for nonlinear wave equations," Advances in Pure Mathematics, vol. 3, pp. 451-457, 2013.

[7] Y. Guo and M. A. Rammaha, "Global existence and decay of energy to systems of wave equations with damping and supercritical sources," Zeitschrift für Angewandte Mathematik und Physik, vol. 64, no. 3, pp. 621-658, 2013.

[8] L. Bociu, M. Rammaha, and D. Toundykov, "On a wave equation with supercritical interior and boundary sources and damping terms," Mathematische Nachrichten, vol. 284, no. 16, pp. 20322064, 2011. 
[9] C. O. Alves, M. M. Cavalcanti, V. N. Domingos Cavalcanti, M. A. Rammaha, and D. Toundykov, "On existence, uniform decay rates and blow up for solutions of systems of nonlinear wave equations with damping and source terms," Discrete and Continuous Dynamical Systems, vol. 2, no. 3, pp. 583-608, 2009.

[10] M. M. Cavalcanti, V. N. Domingos Cavalcanti, and I. Lasiecka, "Well-posedness and optimal decay rates for the wave equation with nonlinear boundary damping-source interaction," Journal of Differential Equations, vol. 236, no. 2, pp. 407-459, 2007.

[11] C. O. Alves and M. M. Cavalcanti, "On existence, uniform decay rates and blow up for solutions of the 2-D wave equation with exponential source," Calculus of Variations and Partial Differential Equations, vol. 34, no. 3, pp. 377-411, 2009.

[12] M. A. Rammaha, "The influence of damping and source terms on solutions of nonlinear wave equations," Boletim da Sociedade Paranaense de Matemática, vol. 25, no. 1-2, pp. 77-90, 2007.

[13] V. Barbu, I. Lasiecka, and M. A. Rammaha, "Existence and uniqueness of solutions to wave equations with nonlinear degenerate damping and source terms," Control and Cybernetics, vol. 34, no. 3, pp. 665-687, 2005.

[14] M. M. Cavalcanti and V. N. Domingos Cavalcanti, "Existence and asymptotic stability for evolution problems on manifolds with damping and source terms," Journal of Mathematical Analysis and Applications, vol. 291, no. 1, pp. 109-127, 2004.

[15] M. M. Cavalcanti, V. N. D. Cavalcanti, J. S. Prates Filho, and J. A. Soriano, "Existence and uniform decay of solutions of a parabolic-hyperbolic equation with nonlinear boundary damping and boundary source term," Communications in Analysis and Geometry, vol. 10, no. 3, pp. 451-466, 2002. 


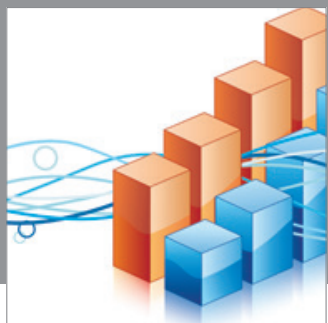

Advances in

Operations Research

mansans

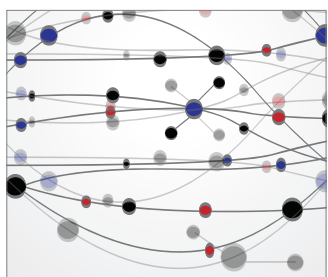

The Scientific World Journal
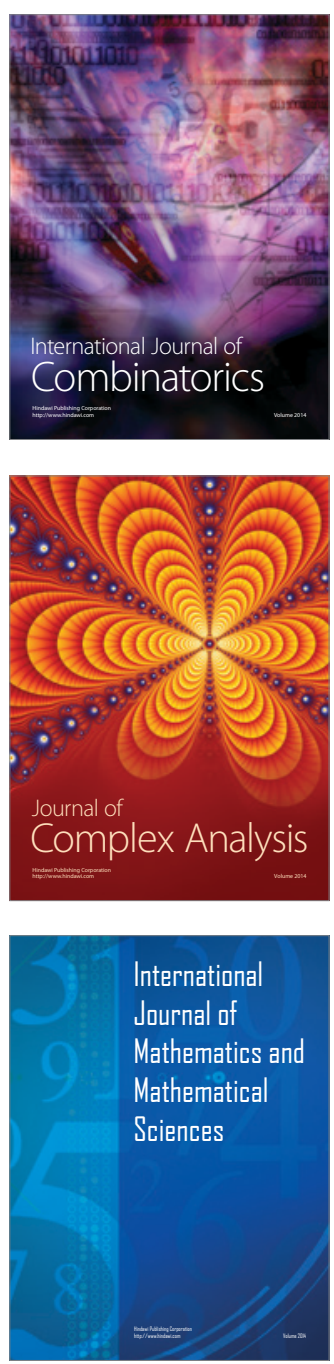
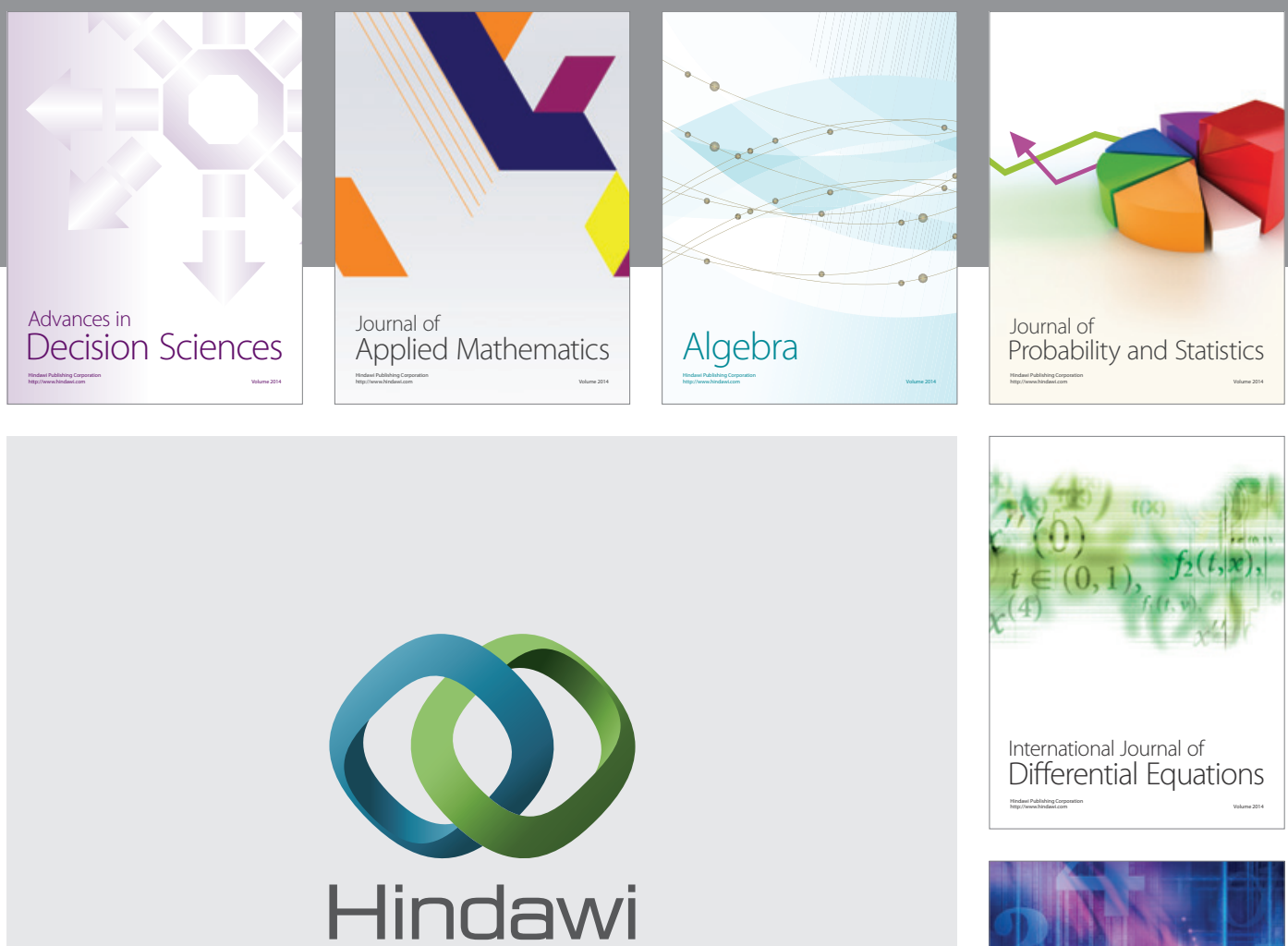

Submit your manuscripts at http://www.hindawi.com
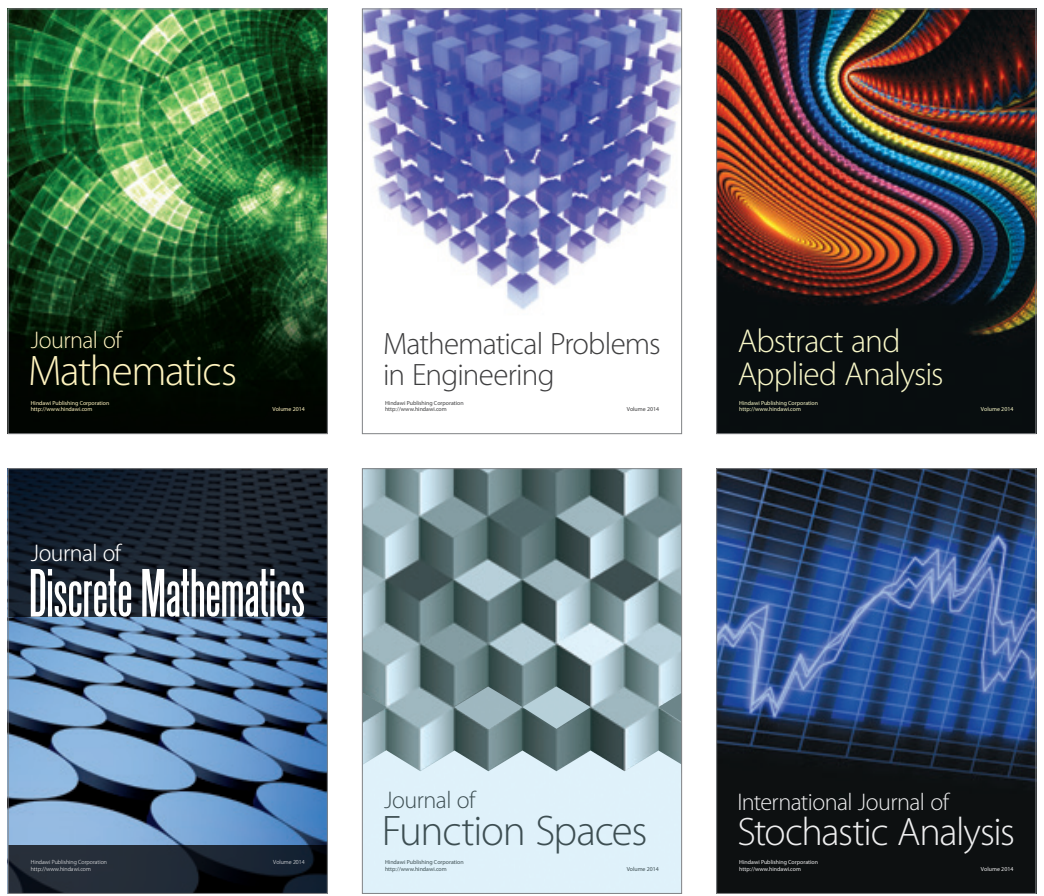

Journal of

Function Spaces

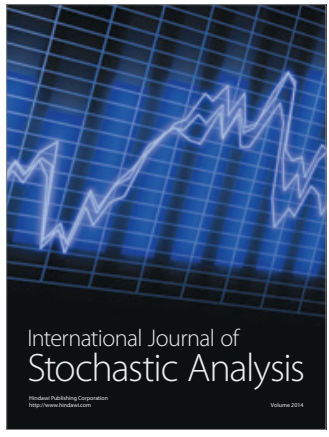

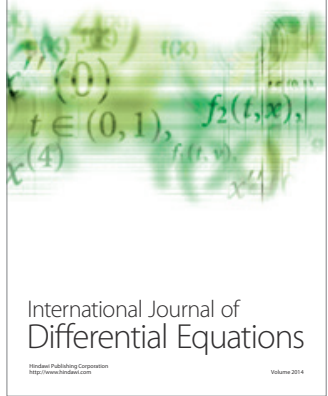
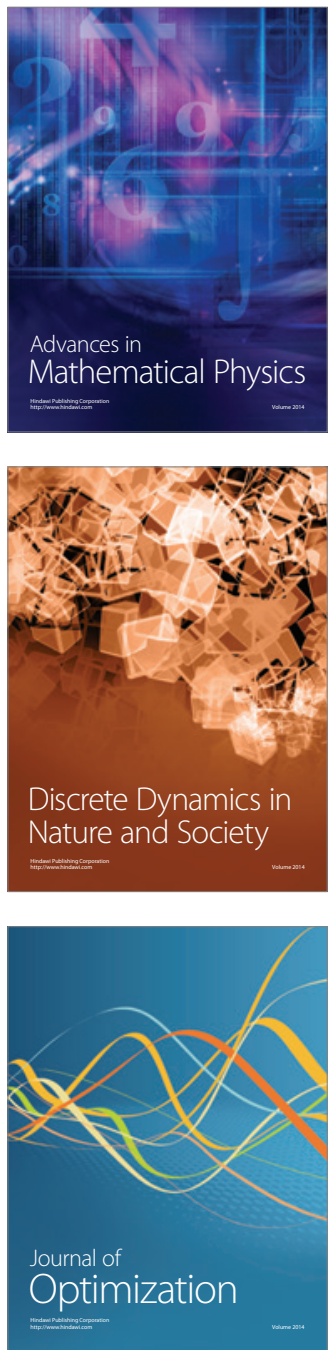